American Indian patients and those identifying as multiple races who have a higher rate $(11.5 \%$ and $10.5 \%)$ than white patients (8.1\%).

Conclusions SPC methodology allows clinicians to use EMR data to understand how patients' race effects their outcomes across a range of acute care domains. Enabling them to track the effect of system changes to understand if equity improves.

\section{REDUCING OPIOID PRESCRIPTIONS FOR PEDIATRIC SUPRACONDYLAR HUMERUS FRACTURES}

Leanne Winslow, Jessica Holstine, Julie Balch Samora. Nationwide Children's Hospital, Columbus, Ohio

\subsection{6/bmjoq-2020-IHI.30}

Background Supracondylar humerus $(\mathrm{SCH})$ fractures are the most common elbow injuries in children. Pain management following treatment is essential, as untreated pain could lead to delayed healing, hyperesthesia, and fear of medical care. Pain management has not been standardized, and variability exists in opioid prescribing patterns. While effective for pain control, opioid therapy has associated adverse events including nausea, vomiting, constipation, blood pressure swings, and physical dependence. As the national opioid crisis continues,

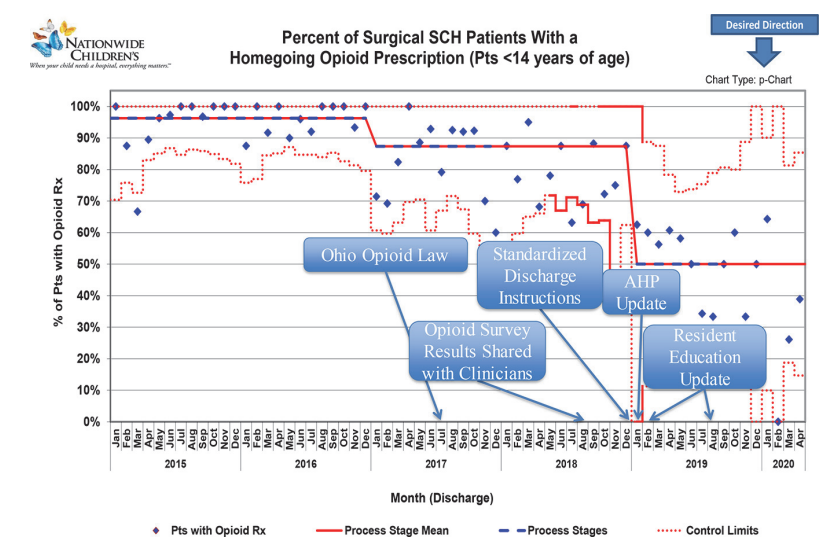

Abstract 30 Figure 1

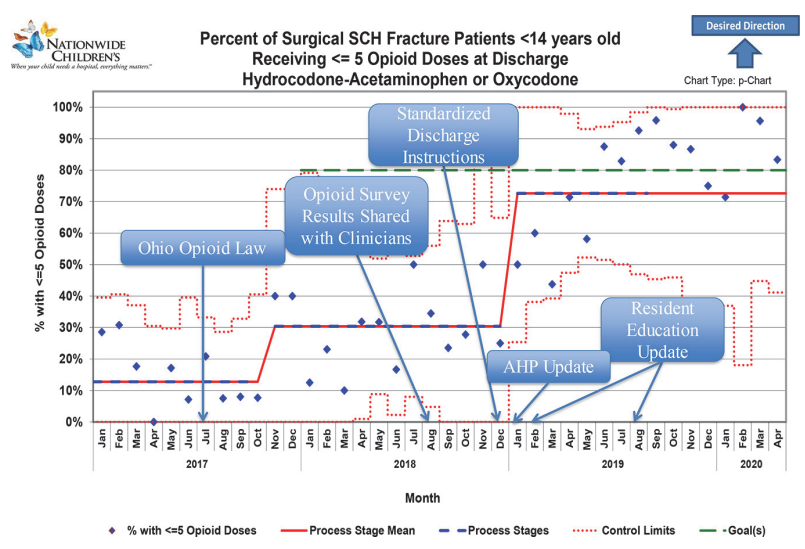

Abstract 30 Figure 2

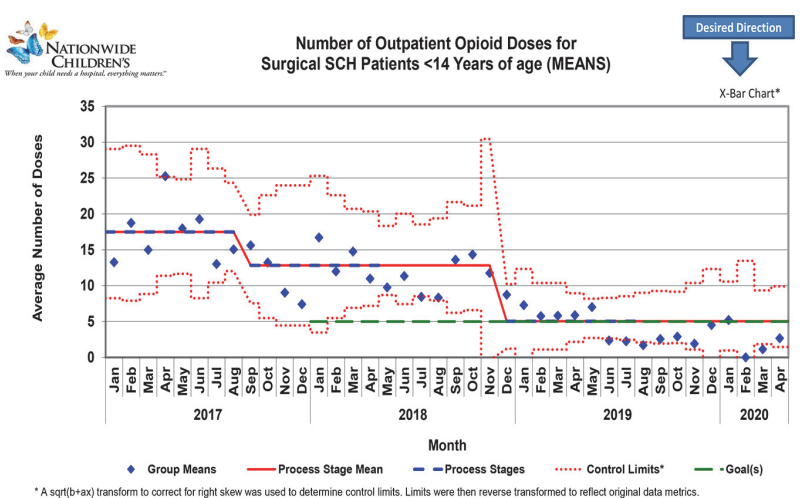

Abstract 30 Figure 3

this project highlights our efforts to reduce the opioid footprint.

Objectives Increase the percent of patients receiving $\leq 5$ doses of hydrocodone-acetaminophen for home use, after a surgical repair of an $\mathrm{SCH}$ fracture, from $30 \%$ to $80 \%$ by $4 / 1 / 19$ and sustain for one year.

Methods A survey was distributed to orthopedic providers as a self-appraisal of opioid prescribing relative to peers. Survey results guided the development of educational interventions. This project standardized the use of non-opioid pain control to limit opioid prescribing. Providers were encouraged to schedule alternating doses of acetaminophen and ibuprofen every three hours. Standard discharge instructions for pain medication paralleled its use during the child's hospitalization. Measures included percent of patients with a homegoing opioid prescription, the number of doses prescribed, and whether the patient called back due to uncontrolled pain.

Results Our orthopedic team increased the percent of opioid prescriptions of $\leq 5$ doses for $\mathrm{SCH}$ fractures from $30 \%$ to $75 \%$ (figure 2). Average prescribed doses decreased from 12 to 5 (figure 3 ). We have reduced the number of patients discharged with an opioid from $96.2 \%$ to $46 \%$ (figure 1).

Conclusions Through this QI initiative, we were able to significantly reduce our opioid prescribing practices for patients with $\mathrm{SCH}$ fractures by establishing prescribing standards, educating providers and standardizing discharge medication instructions. We anticipate an effective translation of these practices to other orthopedic injuries and to other institutions.

\section{READING PROFICIENCY IS A SOCIAL DETERMINANT OF HEALTH: IMPROVING POPULATION OUTCOMES AND REDUCING RACIAL DISPARITIES IN EDUCATION}

Heidi Black, Cheryl Broadnax. StriveTogether, USA

\subsection{6/bmjoq-2020-IHI.31}

Background Spartanburg County, S.C., faces a major economic mobility challenge, particularly for Black and Latinx children and families. Spartanburg leaders were particularly concerned with the low third-grade reading proficiency rates across the 